\title{
Efficient Path Protection using Bi-directional WDM Transmission Technology
}

\author{
Ji Li and Kwan L. Yeung \\ Department of Electrical and Electronic Engineering \\ The University of Hong Kong Hong Kong, PRC \\ Tel: (852) 2857-8493 Fax: (852) 2559-8738 \\ E-mail: \{jili, kyeung\}@eee.hku.hk
}

\begin{abstract}
Bi-directional WDM transmission is a technique that allows wavelengths to be transmitted simultaneously in both directions in a single fiber. Compared with unidirectional WDM systems, it not only saves the cost of deploying extra fibers, but also allows more flexible bandwidth provisioning. To exploit the advantages brought by this flexibility, we investigate path protection based on bi-directional WDM transmission system in this paper. With path protection, a call is accepted if and only if an active data path together with a disjointed backup path can be found in the network. With bi-directional WDM, backup resources sharing in both directions of a fiber is possible. To encourage resources sharing, new cost functions are judiciously designed. Based on them, two original path protection schemes are proposed in this paper, BiPro and BiProLP, where BiProLP aims at further economizing the hardware cost incurred by BiPro. In contrast to the traditional unidirectional schemes, we show that both BiPro and BiProLP can yield noticeably lower call blocking probability, higher system capacity and shorter active/backup path length.
\end{abstract}

\section{INTRODUCTION}

Wavelength-Division Multiplexing [1] (WDM) is a very promising technology to construct optical mesh networks. Exploiting the large bandwidth of optical fibers, WDM couples multiple wavelengths onto one single fiber. In circuit-switch WDM mesh network, a concatenation of wavelengths on different fibers provides a high-speed end-to-end connection called lightpath. To deliver reliable services, WDM optical networks require efficient recovery schemes to protect the traffic carried on different paths. Many schemes have been proposed and studied in the literature [2-10]. They differ from each other in recovery methods adopted and/or routing algorithms used. In this paper, we consider path protection based schemes for its significant capacity savings [10]. With path protection, a call is accepted if and only if an end-to-end active path/lightpath (AP) together with a disjointed backup path/lightpath (BP) can be found in the network. The traffic on the active path is protected by switching data onto BP if some failure occurs on AP.

Different routing algorithms have been proposed for path protection. The routing algorithms in [2][7][10] are based on Integer Linear Programming (ILP) formulation, aiming at minimizing the sum of wavelength costs consumed by the pair of disjointed active and backup paths. An alternative routing approach is two-step restorable routing [6]. By properly exploiting different natures of $\mathrm{AP}$ and $\mathrm{BP}$, two-step routing finds the widest-shortest path for AP and the shortest-widest path for BP. Results in [6] showed that a better performance than ILP based schemes can be achieved. In this paper, we extend the two-step routing to bi-directional path protection.

All previous path protection schemes assumed that WDM transmission mechanism is unidirectional, i.e. at least two fibers have to be provided for duplex transmission between two nodes. The direction and the number of optical channels/wavelengths in each fiber are pre-determined and fixed. With the recent advances in WDM technology, bidirectional WDM system becomes mature and widely available. Bi-directional WDM [1][8] allows any combinations of wavelengths to be transmitted in either direction of a single fiber. Undoubtedly, this can significantly save the cost of deploying a WDM network, as a single fiber spanning between each neighboring node pair is sufficient (for duplex transmission). And more importantly, a bi-directional WDM system enables flexible bandwidth allocation.

To exploit the advantages brought by this flexibility, two original bidirectional path protection schemes are proposed in this paper. They are BiPro and BiProLP, whereas BiProLP aims at further economizing the hardware cost of BiPro. Comparing with the existing schemes based on unidirectional transmission, we show that both BiPro and BiProLP yield noticeably lower call blocking probability, higher system capacity and shorter active/backup path length. Besides, if BiProLP is used, the network has the additional flexibility of trading the performance for less hardware cost.

The rest of the paper is organized as follows. Section II reviews the existing bi-directional WDM technology, and presents our bi-directional path protection scheme (BiPro). Section III proposes the alternative protection scheme, BiProLP. The performance of BiPro and BiProLP is compared with a unidirectional scheme (UniPro) in Section IV. Finally we conclude the paper in Section V.

\section{Bi-directional Path Protection (BiPro)}

\section{A. Bi-directional WDM and Backup Sharing}

Bi-directional WDM transmission system [1] uses part of wavelengths in one fiber for transmitting data in one direction and the rest in the opposite direction. It is most important for such a system to properly separate/isolate the wavelengths running in the opposite directions. As reported in [1], bi-

This work is supported by Hong Kong Research Grant Council Earmarked Grant HKU 7150/04E. 


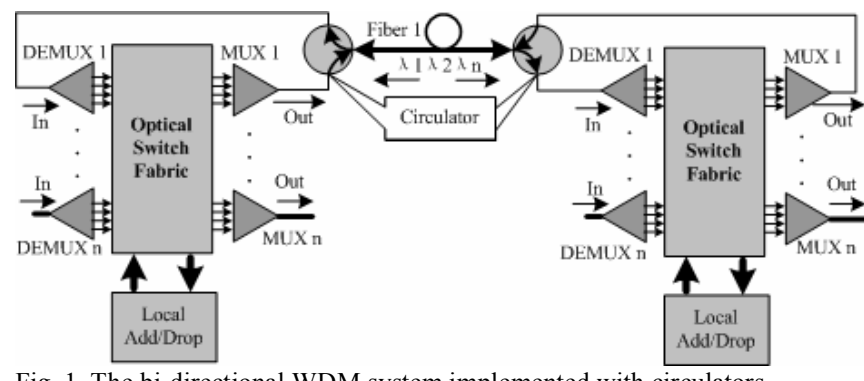

Fig. 1. The bi-directional WDM system implemented with circulators.

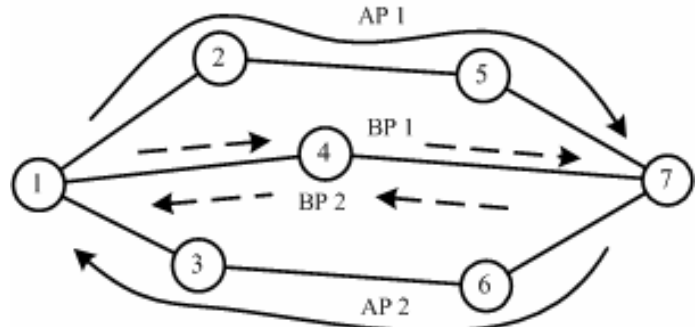

Fig. 2. An example to illustrate bi-directional backup sharing. In unidirectional sharing, four units of bandwidth along BP1 $1 \rightarrow 4 \rightarrow 7$ and BP2 $7 \rightarrow 4 \rightarrow 1$ should be reserved. In BiPro, since the reserved wavelength can be used in either direction, only two units are needed.

directional WDM can be implemented with one of the three separating techniques: band-separation, interleaving and circulator. Without loss of generality, we assume circulator technique is adopted in this paper. A circulator is a multi-port device that allows signals to propagate in certain directions based on the port that the signal came from and blocks all transmission in other directions. Consider an example shown in Fig. 1. Wavelengths arriving at circulator from fiber 1 can only be transmitted toward the input ports of switch, while other wavelengths outputted by switch are guided onto the fiber. Compared with band-separation and interleaving techniques, circulator has the flexibility of supporting asymmetric traffic flows in the same fiber, i.e. arbitrary number of wavelengths in each direction.

If two nodes in a WDM network are directly connected by a link (say link $l$ ), there exist some (say $f_{l}$ ) parallel fibers between them. Assume each fiber can simultaneously carry up to $W$ wavelengths. We call $W$ the capacity of the fiber. The total capacity on link $l$ is thus $f_{l} \cdot W$ wavelengths. This capacity is shared by all the traffic between the two nodes (in both directions). If unidirectional WDM is used, wavelengths inside the same fiber must follow the same direction. Therefore, the wavelengths allocated to the two directions of the link is of the format $\left(x W,\left(f_{l}-x\right) W\right)$, where $x$ is an fixed integer from 0 to $f_{l}$. If bi-directional WDM is adopted, a more flexible bandwidth allocation of $\left(f_{l} W-i, i\right)$ can be obtained, where $i=0, \ldots, f_{l} W$ and can be dynamically adjusted on a call-by-call basis.

Under the assumption that a single network failure occurs at a time, two disjointed active paths (APs) will not be affected by the same link/cable failure, and so their backup paths (BPs) can share the same wavelength. For unidirectional WDM systems, the backup sharing is also unidirectional: a wavelength can only be used to carry those BPs running in the same direction as the fiber it resides in. In bi-directional WDM systems, the reserved backup wavelength can be used to carry the failover traffic in either direction. So two BPs running in the opposite direction can share the same wavelength inside a link, i.e. bi-directional backup sharing. Refer to the example shown in Fig. 2. There are two APs, $1 \rightarrow 2 \rightarrow 5 \rightarrow 7$, and $7 \rightarrow 6 \rightarrow 3 \rightarrow 1$. Each of them requires one wavelength in each traversed link. With unidirectional WDM, the two corresponding backup paths (BP1 \& BP2) are carried by four unidirectional fibers, $1 \rightarrow 4,4 \rightarrow 7,7 \rightarrow 4$ and $4 \rightarrow 1$. So four units of bandwidth (wavelength per fiber) must be reserved. In the case of bi-directional WDM, only two units are needed, in bi-directional fibers 1-4 and 4-7 respectively.

\section{B. Bi-directional Path Protection (BiPro)}

Consider a bi-directional WDM network using three-port circulator (Fig. 1). In order to provide the flexible bandwidth allocation at each node, we need to have $W$ input ports and $W$ output ports for each connected fiber. Assume each node has unlimited local wavelength add/drop capability as well as full wavelength conversion capability. Since cable cut is the most common cause for link failure and generally all the fibers in a link span the same cable, we further assume that parallel fibers between two nodes share the same risk of failure. We also follow the common practice of only considering single failure at a time.

Let each connection request be characterized by a tuple $(s, d$, $w$ ), where $s$ and $d$ are the source and destination nodes, and $w$ is the number of wavelengths (WLs) requested. For a typical link $l$, its bi-directional channel consists of $\mathrm{A}_{l}$ wavelengths occupied by active paths (in both directions), $\mathrm{B}_{l}$ wavelengths taken by backup paths, and $\mathrm{R}_{l}\left(=f_{l} W-\left(\mathrm{A}_{l}+\mathrm{B}_{l}\right)\right)$ residual/idle wavelengths. Both backup wavelength $\left(\mathrm{B}_{l}\right)$ and idle wavelengths can be used to carry calls in either direction.

When a call arrives, two link disjointed paths (AP \& BP) must be found in order to accept the call. To set up an active path via link $l$, we must check the available resources $\mathrm{R}_{l}$. If $\mathrm{R}_{l} \geq$ $w$, the active path can be accepted with a link cost equals to $w$. Otherwise, the cost is infinite and the call is rejected. The total cost of setting up an active path, or its path cost, is the sum of the costs induced at individual links. This cost is minimized if the hop-distance between the source and the destination is minimized.

Assume active path $a$ is found. Next we need to determine the backup path for $a$ on link $l$. In particular, we need to find out $\mathrm{S}_{l}(a)$, the available backup wavelength on link $l . \mathrm{S}_{l}(a)$ consists of two components, $\mathrm{R}_{l}$ residual wavelengths and $\gamma_{l}(a)$ (out of $\mathrm{B}_{l}$ ) backup wavelengths that can be shared to carry this backup path. That is

$$
\mathrm{S}_{l}(a)=\gamma_{l}(a)+\mathrm{R}_{l}
$$

If $\mathrm{S}_{l}(a) \geq w$, the backup path can be set up on link $l$. To encourage backup resources sharing, the associated link cost is defined as follows.

$$
\text { backup link cost }=\left\{\begin{array}{cl}
0 & \text { if } \gamma_{l}(a) \geq w, l \notin a \\
w-\gamma_{l}(a) & \text { if } 0 \leq \gamma_{l}(a)<w, l \notin a \\
\infty & \text { if } S_{l}(a)<w, \text { or } l \in a
\end{array} .\right.
$$




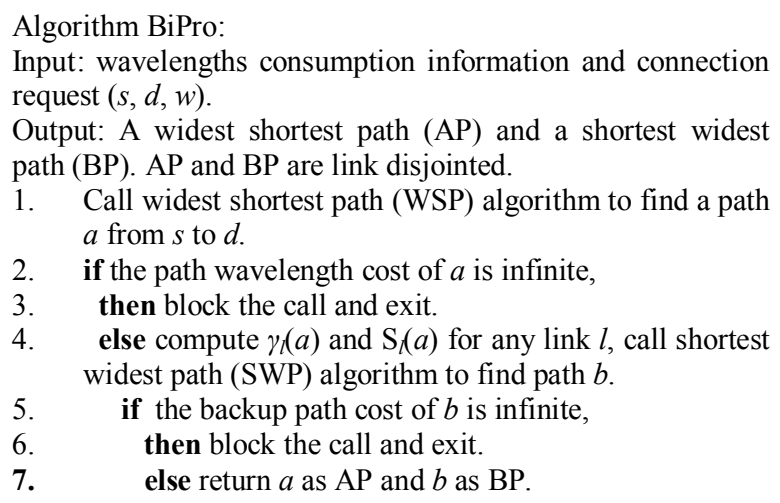

1. Call widest shortest path (WSP) algorithm to find a path $a$ from $s$ to $d$.

2. if the path wavelength cost of $a$ is infinite,

3. then block the call and exit.

4. else compute $\gamma_{l}(a)$ and $\mathrm{S}_{l}(a)$ for any link $l$, call shortest widest path (SWP) algorithm to find path $b$.

5. if the backup path cost of $b$ is infinite,

6. then block the call and exit.

7. else return $a$ as $\mathrm{AP}$ and $b$ as $\mathrm{BP}$.

Fig. 3. The pseudo code of BiPro routing algorithm.

Unlike active paths, the path cost of a longer backup path may be less than that of a shorter one, because of backup sharing.

Next we derive $\gamma_{l}(a)$. Assume link $m$ is traversed by active path $a$. Let $\boldsymbol{\Omega}$ denote the set of active paths carried by link $m$ (including paths running in both directions) and have their backup paths passing through link $l$. Note that the wavelengths reserved by the above backup paths cannot be mutually shared. Assume the number of wavelengths taken up on link $l$ due to active paths in $\Omega$ is $\mathrm{B}_{l}^{m}$. We have

$$
\gamma_{l}(a)=\mathrm{B}_{l}-\max _{m \in a} \mathrm{~B}_{l}^{n} .
$$

The second term $\max _{m \in a} \mathrm{~B}_{l}^{m}$ on the right hand side is to take the maximum of $\mathrm{B}_{l}^{m}$ over all possible links $m$ along the active path $a$, that are protected by the backup paths on link $l$.

Based on the above defined cost functions, a routing algorithm can be used to determine a pair of disjointed AP and BP for a given call. The pseudo code of our proposed routing algorithm (designed based on the two-step routing in [6]) is summarized in Fig. 3. In particular, the widest shortest path algorithm is to find the (active) path that has the widest residual bottleneck capacity $\left(\mathrm{R}_{l}\right)$ among all the shortest paths between the source $(s)$ and the destination $(d)$. If the active path is found successfully, then the shortest widest path algorithm is activated to find the shortest (backup) path among all the widest paths (which have the same widest bottleneck backup cost on $\mathrm{S}_{l}(a)$ ). We call the resulting path protection scheme BiPro.

\section{Bi-Directional Path Protection With Limited Port NUMBER (BIPROLP)}

\section{A. Bi-directional Path Protection with Limited Port Number}

In BiPro, we have assumed that there are $2 W$ ports ( $W$ inputs and $W$ outputs) for each fiber at each node (Fig. 1). This ensures that there are always enough ports at a node to support any bandwidth allocation. This high degree of flexibility is, however, at the expense of high port counts/costs. It is obvious that each (engaged) wavelength (AP or BP) only takes up two ports, one at each end (node) of the fiber. That means on the average, each node needs at most $W$ ports ( $W / 2$ for inputs \& $W / 2$ for outputs) for each fiber.

In this bi-directional path protection with limited port number (BiProLP) scheme, we try to reduce the switch port counts while not sacrificing performance. Let the number of input ports and output ports assigned to each fiber be the same and denoted by $K$. For a link with $f_{l}$ parallel bi-directional fibers, $f_{l} K \times f_{l} K$ ports are needed. Likewise, if the switch is connected with $N$ similar links, the dimension of the switch is $N f_{l} K \times N f_{l} K$. When $K=W$, we have the original BiPro. When $K<W$, a saving in the port count can be achieved, but a call may be blocked due to insufficient ports (in addition to insufficient wavelengths). In any case, $K$ must be larger than $W / 2$, or $(W-2 K)$ wavelengths could never be utilized due to its inherent nature of insufficient ports.

\section{B. Port-pair Cost Function}

BiProLP has less flexibility on capacity allocation than BiPro. At most $f_{l} K$ wavelengths of a link can be assigned to operate in the same direction simultaneously. So with BiProLP, a path (either active or backup) can be set up on link $l$ only if 1 ) link $l$ has sufficient idle/available wavelengths; and 2) the two switches connected by link $l$ have sufficient idle/available input/output port-pair. As a result, BiProLP needs to collect routing information on both wavelength and port consumption. As the link cost function in BiProLP is totally identical to that in BiPro, we only focus on deriving the port cost function.

Fig. 4 shows two switches, $u$ and $v$, are connected by a bidirectional link $l$ with capacity $f_{l} W$. Each switch provides $f_{l} K$ input and output ports dedicated to link $l$ ( $K$ for each parallel fiber). From link $l$ 's perspective, it has $f_{l} K$ port-pairs in each direction between $u$ to $v$. For convenience, we use italic "right" or "left" to indicate the direction $u \rightarrow v$ or $v \rightarrow u$.

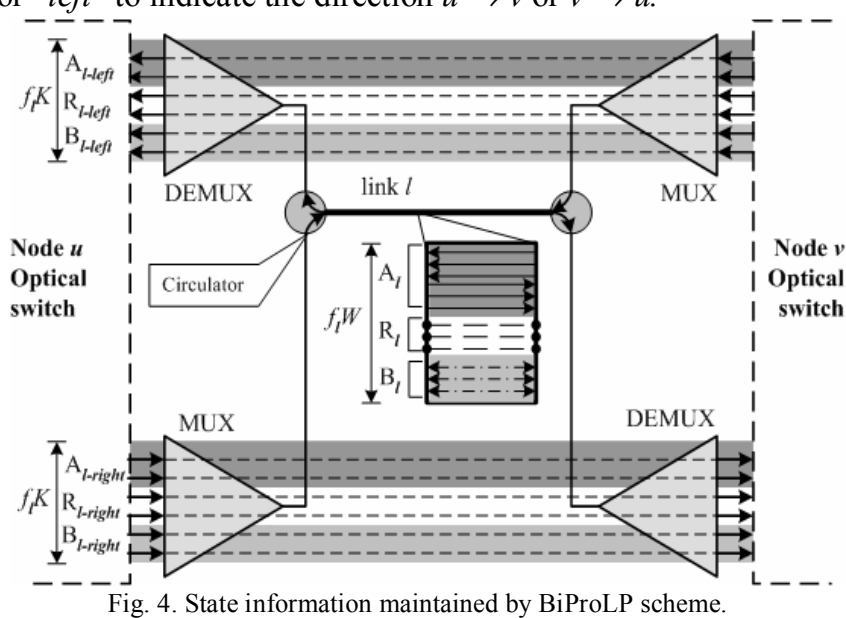

We first derive the port-pair cost functions in the right direction of Fig. 4. (The derivation for the left direction is the same.) Similar to wavelength consumption status, all port-pairs along the right direction can be divided into three parts: $\mathrm{A}_{\text {l-right }}$, $\mathrm{B}_{\text {l-right }}$ and $\mathrm{R}_{\text {l-right }}$. $\mathrm{A}_{\text {l-right }}$ is the total amount of port-pairs occupied by APs through link $l$ (in the right direction). $\mathrm{B}_{l-\text { right }}$ is the total amount of port-pairs reserved for all BPs. Like 


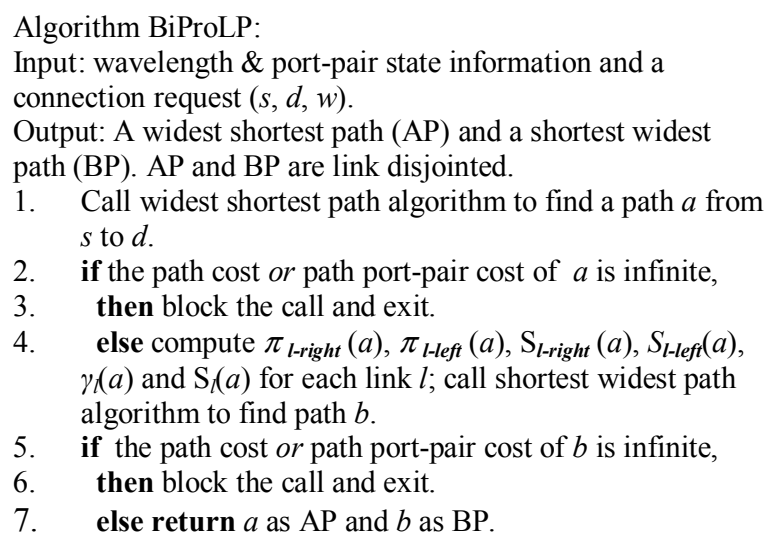

1. Call widest shortest path algorithm to find a path $a$ from $s$ to $d$.

2. if the path cost or path port-pair cost of $a$ is infinite,

3. then block the call and exit.

4. else compute $\pi_{\text {l-right }}(a), \pi_{\text {l-left }}(a), \mathrm{S}_{\text {l-right }}(a), S_{\text {l-left }}(a)$, $\gamma_{l}(a)$ and $\mathrm{S}_{l}(a)$ for each link $l$; call shortest widest path algorithm to find path $b$.

5. if the path cost $o r$ path port-pair cost of $b$ is infinite,

6. then block the call and exit.

7. else return $a$ as $\mathrm{AP}$ and $b$ as $\mathrm{BP}$.

Fig. 5. The pseudo code of BiProLP routing algorithm.

wavelength sharing, the reserved port-pairs on BPs can be shared if the corresponding APs are disjointed. We call it portpair sharing. But port-pair sharing is unidirectional, as port's transmission direction is fixed. So BP1 and BP2 in Fig. 2 cannot share the same port-pair in BiProLP, although they can still share wavelengths. Finally, $\mathrm{R}_{l-\text { right }}\left(=f_{l} K-\left(\mathrm{A}_{l-\text { right }}+\mathrm{B}_{\text {l-right }}\right)\right)$ is the number of residual port-pairs.

To set up an AP via link $l$ from $u \rightarrow v$, we must check both port-availability $\mathrm{R}_{l-\text {-right }}$ and wavelength availability $\mathrm{R}_{l}$. If both are larger than $w$, the active path can be accepted with $w$ as both the link cost and the port-pair cost. Otherwise, the call is rejected. The path port-pair cost is defined as the sum of the port-pair costs on individual links along the selected path.

Considering routing BP on $l$ for AP $a$. Let $\pi_{\text {-right }}(a)$ be the portion of $\mathrm{B}_{\text {l-right }}$ that can be shared by AP $a$. Then $\mathrm{S}_{\text {l-right }}(a)$ the number of available backup port-pairs for carrying $a$ 's BP is given by

$$
\mathrm{S}_{\text {l-right }}(a)=\pi_{\text {-righte }}(a)+\mathrm{R}_{\text {l-right }} .
$$

The link port-pair cost function for backup path setup is

$$
\text { port-pair cost }=\left\{\begin{array}{cl}
0 & \text { if } \pi_{l-r i g h t}(a) \geq w, l \notin a \\
w-\pi_{l-r i g h t}(a) & \text { if } 0 \leq \pi_{l-r i g h t}(a)<w, l \notin a \\
\infty & \text { if } S_{l-r i g h t}(a)<w, \text { or } l \in a
\end{array}\right.
$$

The way to derive $\pi_{l-\text { right }}(a)$ is a little different from deriving $\gamma_{l}(a)$ in equation (3). Assume link $m$ belongs to $a$. Let $\boldsymbol{\Phi}$ denote the set of active paths go through link $m$ 's right port-pairs and have their backup paths passing through right port-pairs of link l. Let the number of APs in set $\Phi$ be $\mathrm{D}_{l-r i g h t}^{m-r i g h t}$. Similarly, we can define $\boldsymbol{\Phi}^{\prime}$ and $\mathrm{D}_{l-\text { right }}^{m \text {-left }}$ for active paths carried by left portpairs on $m$ and have their backup paths passing through right port-pairs on $l$. It should be noticed that the port-pairs reserved for $\boldsymbol{\Phi}$ and $\boldsymbol{\Phi}$ ' cannot be shared.

So we can have

$$
\pi_{\text {-right }}(a)=\mathrm{B}_{\text {l-right }}-\max _{m \in a}\left(\mathrm{D}_{l-\text { right }}^{m-\text { ight }}+\mathrm{D}_{l-\text { right }}^{m \text {-left }}\right) .
$$

The routing algorithm of BiPoLP also follows the two-step routing approach [6], as summarized in Fig. 5.

\section{Simulation Results}

In this section, we compare the performance of our proposed BiPro and BiProLP schemes with a unidirectional protection scheme (UniPro). In UniPro, the same two-step routing algorithm is applied except that the unidirectional WDM transmission is assumed.

The following performance measures are used: call blocking probability, average hop-revenue, active path length, and backup path length. Among them, call blocking probability is the most important measure as it directly reflects the trafficcarrying capability of a network. Average hop-revenue is defined as the average of every admitted call's shortest-hop distance based on the static topology. A higher value indicates that the given algorithm does not jeopardize against long-hop calls. Active path length determines the end-to-end delay performance experienced by the user traffic and backup path length reflects the performance of the user traffic when a network fault occurs.

The simulated network topology is adopted from [2][6]. It has 15 nodes, and 28 links. Each link consists of 2 fibers, i.e. $f_{l}=2$ and each fiber has a capacity of $W=16$. For BiProLP, each fiber has the same number of input/output ports, i.e. $K$. And we tested BiProLP with different $K$ values. We assume a dynamic traffic model that calls arrive at network according to Poisson process with rate $\lambda$. The call holding time follows exponential distribution with $1 / \mu$. The network load is defined as $\lambda \mu$. The source and destination nodes of every call are picked up randomly. The wavelength requirement of each call $w$ is set to 1.

Fig. 6 shows Blocking Probability vs Network Load. We can see that BiPro gives the lowest call blocking probability, whereas UniPro gives the highest. BiProLP (with $K=9$ and 10) are sandwiched by them. The advantage of protections with bidirectional WDM is remarkable, for example, when network load is 160 , the blocking probabilities of UniPro, BiProLP $(K=9)$, BiProLP $(K=10)$, BiPro are respectively $2.14 \%, 1.11 \%$, $0.65 \%, 0.53 \%$. We can see that the UniPro's blocking probability is almost twice as $\operatorname{BiProLP}(K=9)$, more than three times as $\operatorname{BiProLP}(K=10)$ or BiPro. This is due to the flexibility of the bi-directional protection schemes in adapting to asymmetric traffic demands.

From Fig. 6, we can also see the influence of the value $K$ on BiProLP. When the network load is $240, \operatorname{BiProLP}(K=10)$ represents a $37 \%$ drop in $K$ from 16 (i.e. BiPro), The corresponding increase in call blocking is only $3.1 \%$. When $K=9$, the extra increase in blocking probability grows. This shows that with a proper choice of $K$, BiProLP can significantly cut off network hardware cost while keeping a very high overall performance.

Fig. 7 shows Average Hop-Revenue vs Network load. As expected, the average hop-revenue decreases with the load. This is because longer-hop calls are more difficult to be accommodated when resources are tight. However, different schemes have different decreasing rates - UniPro has the 


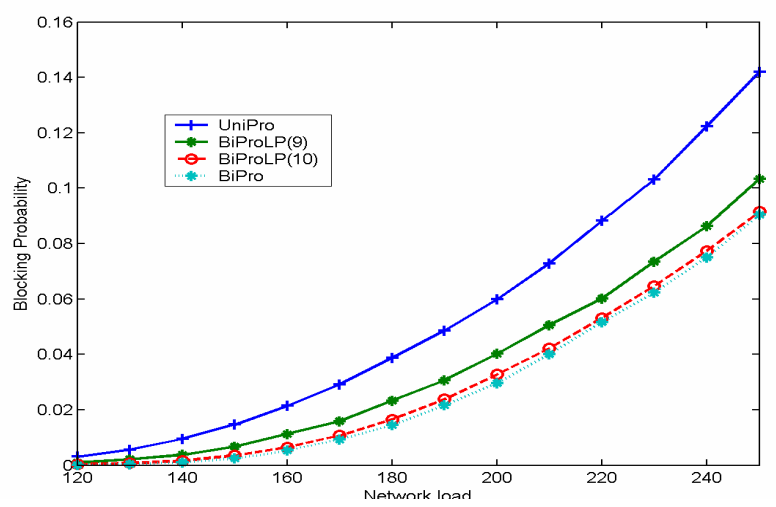

Fig. 6. Blocking Probability vs Network Load.

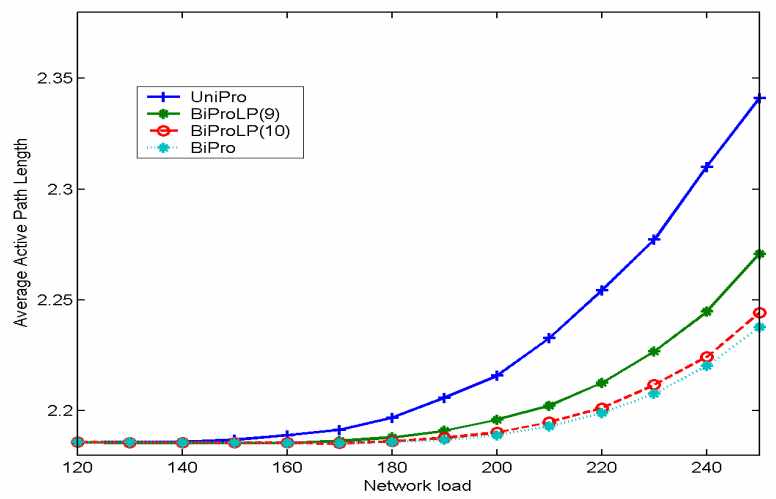

Fig. 8. Average Active Path Length (in hops) vs Network Load.

fastest decreasing rate and BiPro decreases slowest. In general, BiPro gives the highest revenue value, followed by BiProLP $(\mathrm{K}=10)$, BiProLP $(\mathrm{K}=9)$, and UniPro. This clearly indicates that bi-directional path protection schemes are able to provision more long-distance calls than unidirectional schemes.

Fig. 8 and Fig. 9 show Average Active and Backup Path Length (in hops) vs Network Load. We can see that both average active and backup path lengths of BiPro and BiProLP are remarkably shorter than UniPro.

\section{CONCLUSIONS}

In this paper, we investigated path protection based on bidirectional WDM transmission technology. Two original bidirectional protection schemes, BiPro and BiProLP, were proposed to maximize both wavelength and port-pair sharing among backup paths. Due to the flexible bandwidth allocation brought by bi-directional WDM, the network's capability in adapting to time varying traffic distributions has been greatly improved. Comparing with the traditional unidirectional protection scheme, we showed that our schemes yield noticeably lower call blocking probability, higher system capacity, and shorter active/backup path length. With scheme BiProLP, it provides an additional flexibility in lowering the deployment cost while not sacrificing the overall network performance.

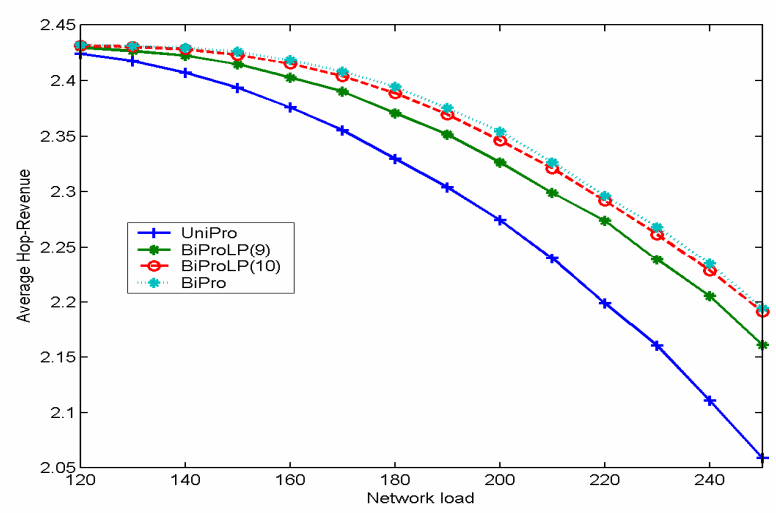

Fig. 7. Average Hop Revenue vs Network Load.

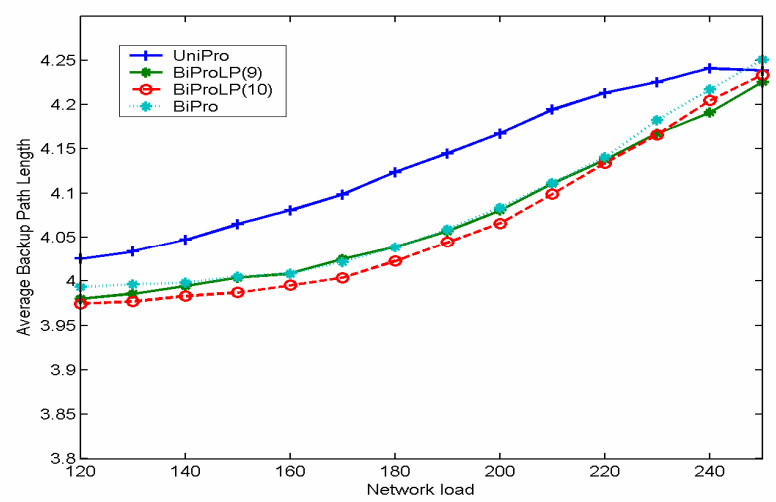

Fig. 9. Average Backup Path Length (in hops) vs Network Load.

[1] V. Alwayn, Optical Network Design and Implmentation, Cisco Press, Indianapolis, 2004.

[2] M. Kodialam and T.V. Lakshman, "Dynamic routing of bandwidth guaranteed tunnels with restoration," in Proc. IEEE INFOCOM 2000, vol. 2, pp. 902-911.

[3] G. Mohan, C. Siva Ram Murthy and A.K. Somani, "Efficient algorithms for routing dependable connections in WDM optical networks," IEEE/ACM Tran. on Networking, Vol. 9, NO. 5, Oct. 2001.

[4] G. Li, D. Wang, C. Kalmanek and R. Doverspike, "Efficient distributed path selection for shared restoration connections," in Proc. IEEE INFOCOM 2002, vol. 1, pp. 140-149, Jun. 2002.

[5] S. Gowda and K. M. Sivalingam, "Protection mechanisms for optical WDM networks based on wavelength converter multiplexing and backup path relocation techniques," in Proc. IEEE INFOCOM 2003, vol. 1, pp. 12-21, Mar. 2003.

[6] J. Li and K. L. Yeung, "A novel two-step approach to restorable dynamic QoS routing", to be appeared in J. Lightwave Technology, Nov. 2005.

[7] Y. Xiong, D. Xu and C. Qiao, "Achieving fast and bandwidth-efficient shared-path protection,” J. Lightwave Technology, vol. 21, pp. 365-371, Feb. 2003.

[8] R. Ramaswami and K. N. Sivarajan, Optical Networks: a Practical Perspective, ver. $2^{\text {nd }}$, Academic Press, San Diego, 2002.

[9] C. Qiao and D. Xu, "Distributed partial information management (DPIM) schemes for survivable networks-part 1," in Proc. IEEE INFOCOM 2002, vol. 1, pp. 302-311, Jun. 2002.

[10] S. Ramamurthy, L. Sahasrabuddhe, B. Mukherjee, "Survivable WDM Mesh Networks," J. Lightwave Technology, vol. 21, pp. 870- 883, Apr. 2003.

\section{REFERENCES}

